\title{
Enfoque multiprofissional na segurança do paciente no ambiente hospitalar: revisão integrativa
}

\author{
Multiprofessional approach to patient safety in the hospital environment: an integrative \\ review
}
Enfoque multiprofesional para la seguridad del paciente en el entorno hospitalario: una revisión integradora

Renata Polyane Araújo Pessoa ${ }^{1}$, Rosane Pereira dos Reis ${ }^{2}$, Douglas Ferreira Rocha Barbosa ${ }^{2 *}$, Guilherme Benjamin Brandão Pitta ${ }^{3}$, Ediane Gonçalves², Rosa Layse Saboya de Melo², Isamara Santos da Silva², Layanne Ramalho Jacob³ ${ }^{3}$, Karla Adriana de Melo², Daniele Gonçalves Bezerra4.

\section{RESUMO}

Objetivo: Descrever através de uma revisão integrativa a segurança do paciente no ambiente hospitalar tendo como enfoque a visão da equipe multiprofissional. Métodos: Trata-se de uma revisão integrativa de literatura, com abordagem qualitativa, onde o levantamento bibliográfico foi realizado no ano de 2019, por meio da consulta direta na internet através dos dados online disponíveis na Biblioteca Virtual de Saúde (BVS), nas seguintes bases de dados Literatura Latino-Americana e do Caribe em Ciências da Saúde (LILACS), Scientific Eletronic Library Online (SciELO), e Base de Dados em Enfermagem (BDENF), totalizando dezesseis artigos para amostra. Resultados: Os resultados evidenciaram que a equipe multiprofissional necessita se instrumentalizar com indicadores que avaliem tanto a estrutura quanto o processo e os resultados de suas ações, buscando estabelecer um olhar mais amplo para a qualidade e segurança da assistência proporcionada. Considerações finais: Concluímos que a segurança do paciente, em todas as suas extensões, vem se tornando uma preocupação constante para o setor da saúde, ensejo pelo qual é um assunto que necessita ser fortemente debatido na atualidade, devido às proeminências do impacto da ocorrência dos erros e dos eventos adversos nos sistemas de saúde.

Palavras-chave: Segurança do paciente, Qualidade da assistência à saúde, Equipe de assistência ao paciente, Hospitalização.

\section{ABSTRACT}

Objective: Describe, through an integrative review, patient safety in the hospital environment, focusing on the vision of the multidisciplinary team. Methods: This is an integrative literature review, with a qualitative approach, where the bibliographic survey was carried out in 2019, through direct consultation on the internet through online data available at the Virtual Health Library (VHL), in the following databases Latin American and Caribbean Literature on Health Sciences (LILACS), Scientific Electronic Library Online (SciELO), and Nursing Database (BDENF), totaling sixteen articles for sample. Results: The results showed that the multiprofessional team needs to be equipped with indicators that assess both the structure and the process and the results of their actions, seeking to establish a broader look at the quality and safety of the care

\footnotetext{
${ }^{1}$ Hospital da Mulher Dr ${ }^{a}$ Nise da Silveira, Maceió - Alagoas.

*E-mail: dougrochanz@gmail.com

2 Faculdade Estácio de Alagoas, Maceió - Alagoas.

${ }^{3}$ Universidade Estadual de Ciências da Saúde de Alagoas (UNCISAL), Maceió - Alagoas

${ }^{4}$ Universidade Federal de Alagoas (UFAL), Maceió - Alagoas.
} 
provided. Final considerations: We conclude that patient safety, in all its extensions, has become a constant concern for the health sector, an opportunity for which it is a subject that needs to be strongly debated today, due to the prominence of the impact of the occurrence of errors and errors, adverse events in health systems.

Key words: Patient safety, Quality of health care, Patient care team, Hospitalization.

\section{RESUMEN}

Objetivo: Describir, a través de una revisión integradora, la seguridad del paciente en el entorno hospitalario, centrándose en la visión del equipo multiprofesional. Métodos: Esta es una revisión bibliográfica integradora, con un enfoque cualitativo, donde la encuesta bibliográfica se realizó en 2019, a través de consultas directas en Internet a través de datos en línea disponibles en la Biblioteca Virtual en Salud (BVS), en las siguientes bases de datos. Literatura latinoamericana y caribeña sobre ciencias de la salud (LILACS), Biblioteca electrónica científica en línea (SciELO) y Base de datos de enfermería (BDENF), con un total de dieciséis artículos por muestra. Resultados: Los resultados mostraron que el equipo multiprofesional debe estar equipado con indicadores que evalúen tanto la estructura como el proceso y los resultados de sus acciones, buscando establecer una visión más amplia de la calidad y seguridad de la atención brindada. Consideraciones finales: Concluimos que la seguridad del paciente, en todas sus extensiones, se ha convertido en una preocupación constante para el sector de la salud, una oportunidad por la cual es un tema que debe debatirse enérgicamente hoy, debido a la importancia del impacto de la ocurrencia de errores y errores, eventos adversos en los sistemas de salud.

Palabras clave: Seguridad del paciente, Calidad de la atención médica, Equipo de atención al paciente, Hospitalización.

\section{INTRODUÇÃO}

Nos últimos anos a segurança do paciente tem se tornado um dos assuntos prioritários na área da saúde. É uma das principais finalidades intensamente desejada pelas instituições de saúde que procuram garantir uma assistência de qualidade, livre de falhas e eventos adversos. É dever dos profissionais de saúde proporcionar uma assistência de qualidade, hábil, dinâmica e segura ao paciente (CAVALCANTE AKCB, et al., 2015). Segundo Silva ACA, et al. (2016), as questões integradas à segurança do paciente constituem em um grave problema de saúde em todo o mundo.

Nesse sentido, os riscos e os episódios de eventos adversos que provocam agravos ao paciente, têm aumentado em todos os ambientes, em especial no âmbito hospitalar. No entanto, tem se notado uma preocupação em torno das políticas para a melhoria da qualidade assistencial, e a problemática que envolve os riscos à segurança do paciente se torna questão de diversos estudos no sistema de saúde.

O ambiente hospitalar exibe numerosos riscos à saúde dos pacientes, os quais podem piorar o seu estado de saúde. Deste modo, incumbe aos profissionais analisar os riscos à saúde presentes em cada setor, a fim de assegurar a segurança dos pacientes e o restabelecimento de sua saúde, bem como impedir ou tornar mínimo as intercorrências durante sua estada na instituição. O cuidado precisa ser realizado sem ocasionar agravo, de modo a permear a integralidade da assistência (SILVA AT, et al., 2018).

Diante desse contexto, cabe salientar que a segurança do paciente é influenciada, embora os progressos na área de saúde, pelas doenças ocasionadas por erros médicos acometidos pelos profissionais, as quais refletem espontaneamente na qualidade de vida dos pacientes, gerando implicações desagradáveis tanto para os pacientes como para os profissionais e para a organização hospitalar (SILVA AT, et al., 2016). É importante ressaltar que são crescentes as ações direcionadas para a promoção da segurança e da qualidade na assistência à saúde em âmbito mundial, com envolvimento da alta chefia das instituições até seus colaboradores. Como implicação, a meta de qualidade nos diversos serviços proporcionados à sociedade implica a otimização dos resultados (OLIVEIRA RM, et al., 2014). 
"No Brasil, o Ministério da Saúde (MS) instituiu, em 2013, o Programa Nacional de Segurança do Paciente (PNSP) com a finalidade de implementar medidas assistenciais, educativas e programáticas e iniciativas voltadas à segurança do paciente em diversas áreas da atenção, organização e gestão de serviços de saúde por meio da implantação da gestão de risco e de Núcleos de Segurança do Paciente nos estabelecimentos de saúde" (BRASIL, 2014).

Para tanto, a Organização Mundial de Saúde (OMS) recomendou que gestores considerassem as perspectivas dos cidadãos na tomada de decisão, determinando que a segurança do paciente seja capaz de ser alcançada através de três ações complementares: impedir o acontecimento dos eventos adversos, tornálos sólidos se acontecerem e tornarem mínimo seus efeitos com intervenções dinâmicas (CAVALCANTE AKCB, et al., 2015) e (GRIGOLETO ARL, et al., 2011).

Segundo Nunes FDO, et al., (2014), a incidência mundial de eventos adversos é muito elevada, seu estudo observou que dentre os países estudados, constata-se que 2,9\% a 16,6\% dos pacientes já foram vítimas de eventos adversos, sendo os mesmos suscetíveis de prevenção. Em um estudo realizado no Brasil em um hospital universitário, constatou que $50 \%$ dos pacientes em alta hospitalar e $70 \%$ dos pacientes que evoluíram a óbitos, tiveram pelo menos um evento adverso.

De acordo com Pedro DRC, et al. (2016), quando o paciente possui escasso conhecimento sobre seu(s) problema(s) de saúde, pode demonstrar problemas em entender e aceitar a terapêutica e, no caso do acolhimento hospitalar, prolongando sua internação e provavelmente aumentando riscos à sua saúde. Por esse motivo, inclusive, faz-se necessário que os profissionais de saúde pratiquem a comunicação entre equipe e com os pacientes, porque isso pode promover o processo de trabalho e diminuir os riscos inerentes à assistência, colaborando para o cuidado seguro.

Ainda que as diferentes ações recomendadas para melhorar a segurança nos hospitais incluam instrutores e administradores hospitalares, entre outros profissionais, ao mesmo tempo, é indispensável à atuação da equipe de multiprofissional, para garantir o cuidado confiável e qualificado, essencial nessa área (MÍRANDA AP, et al., 2017).

$\mathrm{Na}$ área da saúde, que se constitui num peculiar setor de prestação de serviços, a comunicação tem potencial para auxiliar o cuidado absoluto e humanizado, e por isso, infere-se que este é um fator que merece atenção à promoção do acolhimento garantido em todos os níveis de atenção (WHITE GD, et al., 2015). Isso porque, falhas nas linhas de comunicação podem beneficiar a episódio de eventos adversos (MELLO JF e BARBOSA SFF, 2013), que são definidos como incidentes ocasionados pelo processo de cuidado que derivam em dano fidedigno, de magnitude variada, à saúde do usuário (BRASIL, 2014).

Alguns autores ressaltaram que principalmente no contexto hospitalar, em que o risco associado à assistência é mais perceptível, as linhas de comunicação entre profissionais e/ou setores podem ser um componente de dificuldade à sua eficácia (MELLO JF e BARBOSA SFF, 2013). Isso provavelmente é influenciado pelo modelo de gestão e da cultura organizacional vigente, o que pode desdobrar-se nas práticas de (in)segurança do paciente (BOHRER CD, et al., 2016).

Considerando que no processo de acolhimento à saúde o risco de o paciente sofrer danos é maior quando os processos institucionais não são esquematizados ou são esquematizados de forma imprópria, este estudo se justifica porque é de suma importância o desvelamento e a divulgação de informações que cooperem para a prevenção de danos ao paciente, especialmente quando se refere ao processo de implantação de estratégias voltadas à segurança (REIS GAX, et al., 2017). Diante do explicitado, acredita-se que ultimamente, na área da saúde, há uma enorme busca pela qualidade e pela segurança, amparada por políticas públicas, originando alterações expressivas no Sistema Único de Saúde (SUS) e nos modelos de gestão das instituições. Por sua vez, as alterações demográficas, sociais e econômicas que a sociedade brasileira vivencia, ultimamente, têm impactado as categorias de vida e saúde da população e, portanto, colaboram com novas ações para o sistema e serviços de saúde do país, influenciando a adaptação a novos perfis de necessidades (ARAÚJO MAN, et al., 2017). 
Com base no explanado anterior questiona-se: "Quais as evidências científicas na literatura científica a respeito da segurança do paciente no ambiente hospitalar tendo como enfoque a visão da equipe multiprofissional"? Para responder a esta indagação, estabeleceu-se como objetivo desta investigação foi descrever por meio uma revisão integrativa sobre a segurança do paciente no ambiente hospitalar tendo como enfoque a visão da equipe multiprofissional.

\section{MÉTODOS}

O presente estudo trata-se de uma revisão integrativa de literatura, com abordagem qualitativa. $O$ levantamento bibliográfico foi realizado no ano de 2019, por meio da consulta direta na internet através dos dados online disponíveis na Biblioteca Virtual de Saúde (BVS), nas seguintes bases de dados Literatura Latino-Americana e do Caribe em Ciências da Saúde (LILACS), Scientific Eletronic Library Online (SciELO), e Base de Dados em Enfermagem (BDENF).

Assim, inicialmente foi realizada uma busca de artigos que teriam a aplicação desse teste, de maneira geral, em pacientes com as mais diversas patologias, tendo como objetivo identificar as concepções acerca da temática e os possíveis resultados encontrados.

Na busca inicial foram considerados os títulos e os resumos dos artigos para a seleção ampla de prováveis trabalhos de interesse, sendo destacados os resumos (dos artigos que não tinham texto acessível) e os textos completos dos artigos, utilizando-se os seguintes descritores em Ciências da Saúde (DeCS): segurança do paciente, qualidade da assistência à saúde, equipe de assistência ao paciente e hospitalização.

Utilizou-se como critério de inclusão artigos na íntegra que retratem a temática pesquisada, publicados e indexados nos referidos bancos de dados, dos últimos 6 anos (2014 a 2019), português e/ou inglês e espanhol, em caso de títulos repetidos, apenas um deles foi estabelecido e disponível gratuitamente. Também foram utilizadas referências encontradas nos artigos selecionados, devido à importância de seu conteúdo para o presente estudo. Como critério de exclusão, optou-se por não utilizar artigos que não correspondiam ao objeto de estudo, textos que se encontravam incompletos, textos duplicados e que não eram considerados pesquisas ou artigos, assim como é mostrado no Gráfico 1. 
Gráfico 1 - Métodos de inclusão e exclusão utilizadas para a seleção de artigos para o estudo.

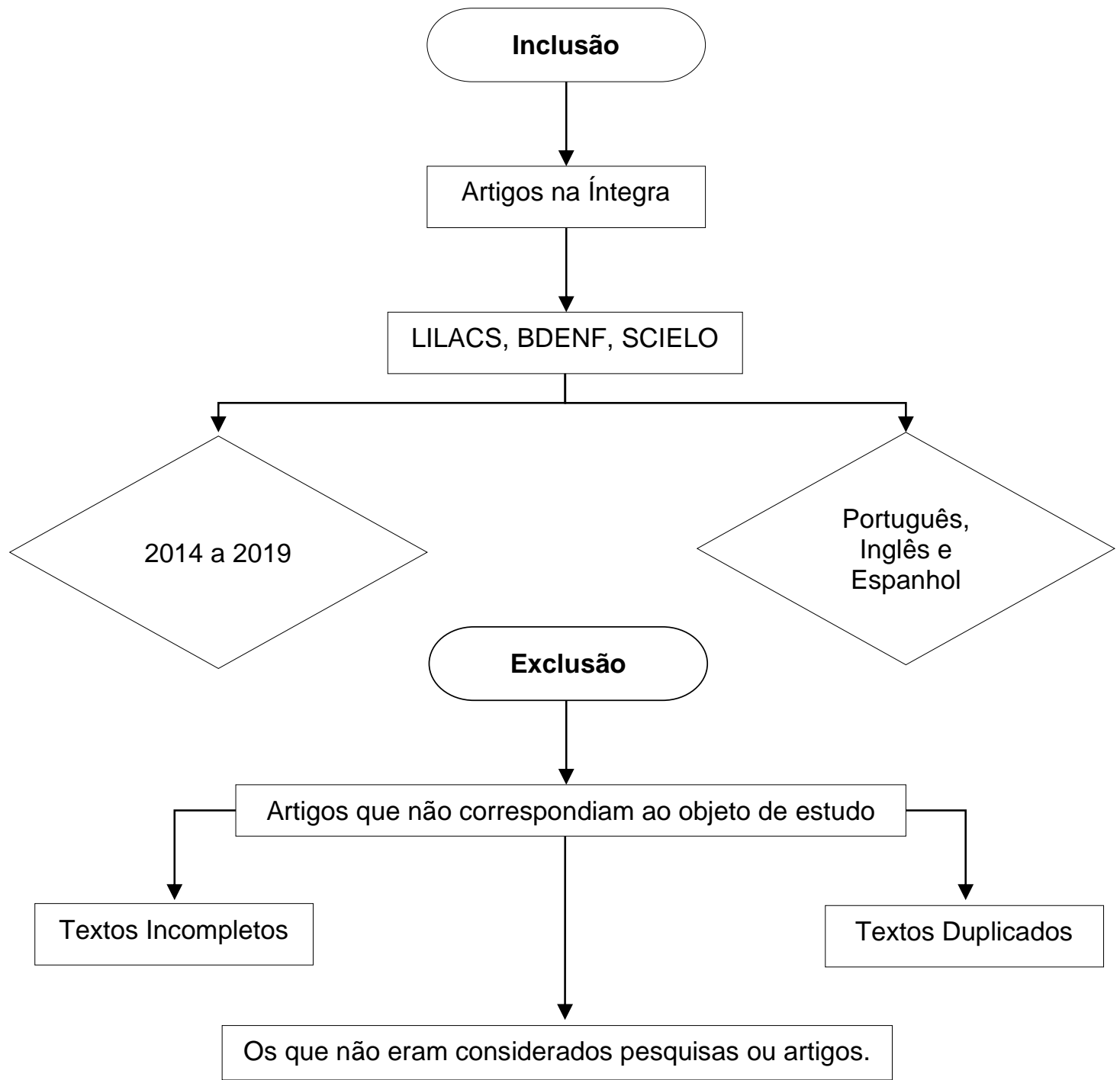

Fonte: Pessoa RPA, et al., 2020.

A seleção dos estudos foi realizada de forma independente, realizado o cruzamento dos descritores utilizando operador booleano AND, por ser o único a mostrar uma busca precisa acerca do foco da temática, com isso sendo possível encontrar os artigos para a realização do estudo.

\section{RESULTADOS}

A aplicação dos critérios de inclusão resultou em 990 artigos, dos quais 974 foram excluídos por fugirem o foco do trabalho resultando em uma amostra filtrada de 16 publicações cuja evolução anual apresenta-se descrita na Tabela 1. 
Tabela 1 - Distribuição das referências selecionadas de acordo com o ano em que foram publicadas. Maceió - AL, $2020(n=16)$.

\begin{tabular}{ccc}
\hline Ano & $\begin{array}{c}\text { Referências } \\
\text { Selecionadas }\end{array}$ & $\begin{array}{c}\text { Porcentagem } \\
(\%)\end{array}$ \\
\hline 2014 & 2 & 12,5 \\
2015 & 1 & 6,25 \\
2016 & 2 & 12,5 \\
2017 & 3 & 18,75 \\
2018 & 5 & 31,25 \\
2019 & 3 & 18,75 \\
\hline Total & 16 & $100 \%$ \\
\hline
\end{tabular}

Fonte: Pessoa RPA, et al., 2020.

A Tabela 2 destaca que na base de dados LILACS obteve-se o maior número de referências e de referências selecionadas para a composição do corpo da pesquisa, seguido pela BDENF e SciELO.

Tabela 2 - Distribuição das referências obtidas nas bases de dados LILACS, BDENF e SciELO. Maceió - AL, $2020(n=16)$.

\begin{tabular}{cccc}
\hline Bases de dados & Referências obtidas & $\begin{array}{c}\text { Referências } \\
\text { selecionadas }\end{array}$ & Porcentagem total (\%) \\
\hline LILACS & 586 & 7 & 43,75 \\
BDENF & 404 & 5 & 31,25 \\
SciELO & 227 & 4 & 25 \\
\hline Total & 990 & 16 & $100 \%$ \\
\hline
\end{tabular}

Fonte: Pessoa RPA, et al., 2020.

Na presente revisão integrativa onze artigos atenderam rigorosamente à seleção da amostra previamente estabelecida. No Quadro 1 abaixo apresenta os artigos selecionados em ordem cronológica de publicação. No que concerne às revistas que obtiveram mais publicações foi possível observar uma diversidade de periódicos que publicaram artigos sobre a temática. 
Quadro 1 - Artigos científicos selecionados nas bases de dados LILACS, SCIELO e BDENF segundo o título, periódico e ano. Maceió, 2020.

\begin{tabular}{|c|c|c|c|c|}
\hline № & Bases de dados & Título do artigo & Periódico/ Ano & Referência \\
\hline 1 & \multirow{7}{*}{ LILACS } & Segurança do paciente na UTI: uma revisão literatura. & Revista Científica FacMais/ 2018. & Da Cruz FF, et al. (2018) \\
\hline 2 & & Risco de quedas em pessoas idosas hospitalizadas. & $\begin{array}{l}\text { Revista Gaúcha de Enfermagem/ } \\
2019 .\end{array}$ & Falcão RMM, et al. (2019) \\
\hline 3 & & $\begin{array}{l}\text { A cultura de segurança do paciente no âmbito da enfermagem: } \\
\text { reflexão teórica. }\end{array}$ & $\begin{array}{l}\text { Revista de Enfermagem do Centro- } \\
\text { Oeste Mineiro /2018. }\end{array}$ & Lemos GC, et al. (2018) \\
\hline 4 & & $\begin{array}{c}\text { O uso de protocolos de segurança do paciente nas instituições } \\
\text { hospitalares. }\end{array}$ & Rev. Bras. Pesq. Saúde/2017. & Souza LO, et al. (2017) \\
\hline 5 & & $\begin{array}{l}\text { A segurança do paciente em âmbito hospitalar: revisão integrativa da } \\
\text { literatura. }\end{array}$ & Cogitare Enfermagem/ 2016. & Silva ACA, et al. (2016) \\
\hline 6 & & $\begin{array}{c}\text { Cultura de Segurança do paciente em instituições de saúde: um } \\
\text { estudo bibliométrico. }\end{array}$ & Enfermería Global/2014. & Tobias GC, et al. (2014) \\
\hline 7 & & $\begin{array}{l}\text { Condutas de educação ao familiar para promoção da segurança da } \\
\text { criança hospitalizada: registros da equipe multiprofissional. }\end{array}$ & $\begin{array}{l}\text { Revista Mineira de } \\
\text { enfermagem/2017. }\end{array}$ & Bandeira LE, et al. (2017) \\
\hline 8 & \multirow{5}{*}{ BDENF } & Cultura de segurança do paciente: avaliação de enfermeiros. & Revista RENE/2019. & Pagani S, et al. (2019) \\
\hline 9 & & $\begin{array}{c}\text { Percepção de familiares e cuidadores quanto à segurança do } \\
\text { paciente em unidades de } \\
\text { internação pediátrica }\end{array}$ & $\begin{array}{l}\text { Revista Gaúcha de } \\
\text { Enfermagem/2018. }\end{array}$ & Peres MA, et al. (2018) \\
\hline 10 & & Fatores extrínsecos para risco de quedas de idosos hospitalizados. & $\begin{array}{l}\text { Revista enfermagem UFPE on } \\
\text { line/2018. }\end{array}$ & Oliveira JS, et al. (2018) \\
\hline 11 & & Avaliação do risco de quedas em idosos hospitalizados. & $\begin{array}{l}\text { Revista enfermagem UFPE on } \\
\text { line/2018. }\end{array}$ & Falcão RMM, et al. (2018) \\
\hline 12 & & Avaliação da implantação de um núcleo de segurança do paciente. & $\begin{array}{l}\text { Revista enfermagem UFPE on } \\
\text { line/2019. }\end{array}$ & Santos RP, et al. (2019) \\
\hline 13 & \multirow{4}{*}{ SCIELO } & Eventos adversos e segurança na assistência de enfermagem. & $\begin{array}{l}\text { Revista Brasileira de } \\
\text { enfermagem/2015. }\end{array}$ & Duarte SCM, et al. (2015) \\
\hline 14 & & $\begin{array}{c}\text { Cultura de segurança do paciente em instituições hospitalares na } \\
\text { perspectiva da enfermagem. }\end{array}$ & $\begin{array}{l}\text { Revista Gaúcha de } \\
\text { Enfermagem/2016. }\end{array}$ & Toso GL, et al. (2016) \\
\hline 15 & & $\begin{array}{c}\text { Segurança do paciente na visão de enfermeiros: uma questão } \\
\text { multiprofissional. }\end{array}$ & Enfermagem em FOCO/2017. & Araújo MAN, et al. (2017) \\
\hline 16 & & $\begin{array}{l}\text { Estratégias para promover segurança do paciente: da identificação } \\
\text { dos riscos às práticas baseadas em evidências. }\end{array}$ & $\begin{array}{l}\text { Escola Anna Nery Revista de } \\
\text { Enfermagem/2014. }\end{array}$ & Oliveira RM, et al. (2014) \\
\hline
\end{tabular}

Fonte: Pessoa RPA, et al., 2020. 


\section{DISCUSSÃO}

Dente os discursos analisados entende-se que amplas alterações têm ocorrido nas últimas décadas para segurança do paciente, criando-se diversos programas e estratégias que estão proporcionando mais segurança ao paciente. Está, também, em ascensão, a construção de uma cultura de segurança permitindo as instituições de saúde e aos profissionais disporem de subsídios e utensílios em prol de uma assistência com qualidade. A segurança do paciente tem sido avaliada como um componente prioritário da qualidade dos serviços de saúde em todo o mundo. Os países da América Latina, inclusive, o Brasil, vêm tornando mais robustas as defesas do sistema de saúde por completo, identificando os melhores utensílios de prevenção a serem aplicados (NASCIMENTO JC e DRAGANOV PB, 2015).

Diante desse contexto, percebe-se que a segurança do paciente depende da adoção de táticas que visam impedir a episódio de eventos adversos preveníveis e, quando não é possível, tornar mínimo suas implicações para o paciente.

Essa abordagem recomenda a adoção de uma cultura de segurança, na qual as falhas possam ser adotadas e evitadas, impulsionando uma prática assistencial mais segura (PERES MA, et al., 2018), pois a segurança tornou-se um elemento eficaz da qualidade do cuidado.

No entanto Toso GL, et al. (2016) entende-se por a segurança do paciente a diminuição ao mínimo aceitável do risco de danos supérfluos, durante a atenção à saúde. Essas táticas procuram impedir, prevenir e tornar mínimo os resultados provenientes de eventos adversos decorrentes das práticas de atenção em saúde associadas ao cuidado. Os eventos adversos são agravos causados pelo cuidado do profissional da saúde por fatores não relacionados à enfermidade de base do paciente, podendo prolongar a hospitalização ou modificar o tratamento recomendado inicialmente (OLIVEIRA JC, et al., 2018).

O crescente número de casos documentados sobre eventos adversos no cuidado à saúde tem provocado discussões sobre a segurança do paciente em âmbito internacional e nacional. A alta complexidade que agrega o ambiente hospitalar é alvo de diversos eventos, o que expõe o paciente ao risco, aumentando a probabilidade da ocorrência de um incidente durante a assistência em saúde (FALCÃO RMM, et al., 2018).

Cabe destacar que dentre as estratégias do Programa Nacional de Segurança do Paciente (PNSP), incluem-se os seis protocolos básicos, definidos pela OMS, integrantes do Programa Nacional de Segurança do Paciente: identificação do paciente; cirurgia segura; prevenção de úlceras por pressão; prática de higiene das mãos em serviços de saúde; segurança na prescrição, uso e administração de medicamentos; prevenção de quedas (BRASIL, 2013).

Destaca-se também que os protocolos fazem parte do PNSP, cuja implantação, no Brasil, seguiu tendência mundial de promoção da segurança do paciente e, em âmbito nacional, houve a melhora da qualidade da Rede de Atenção à Saúde e, por conseguinte, a melhora do Sistema Único de Saúde, principalmente, pelo uso dos fluxos, procedimentos e indicadores propostos para cada processo (SANTOS RP, et al., 2019).

Segundo Souza LO, et al. (2017), adesão aos protocolos propostos pela OMS ainda não é uma realidade nas instituições hospitalares, atribuindo-se como causas as fragilidades pautadas à cultura de segurança nas instituições de saúde, à capacitação profissional; ao pouco engajamento pessoal e coletivo, no que tange à comunicação na equipe de saúde para disseminação e incorporação coletiva da prática assistencial segura e de qualidade.

Em virtude dos avanços tecnológicos, os hospitais tornaram-se cada vez mais um ambiente inseguro e o despreparo de profissionais de saúde pode induzir a falha na prestação da assistência, ocasionando danos de curto, médio ou longo prazo. Ao mesmo tempo, hospitais lotados, escassez de leitos e de profissionais, dupla jornada de trabalho, falta de recursos materiais, dentre outros, elevam o risco de eventos adversos, comprometendo a segurança do paciente e, em alguns casos, provocando até sua morte. Deste modo, a equipe multiprofissional necessita se instrumentalizar com indicadores que avaliem tanto a estrutura quanto o processo e os resultados de suas ações, buscando estabelecer um olhar mais amplo para a qualidade e segurança da assistência proporcionada (ARAÚJO MAN, et al., 2017). 
Duarte SCM, et al. (2015), ressalta que apesar de todos os avanços no âmbito da segurança do paciente, o erro humano é um dos fatores que se destaca e repetidamente os casos de erro envolvendo profissionais de saúde nas instituições hospitalares são noticiados através da imprensa e da mídia, ocasionando ampla comoção social.

Vale ressalvar que a ausência de conhecimento e segurança dos profissionais de saúde, fazem com que acabem realizando procedimentos errados, colocando em risco a vida do paciente. Por esse motivo garantir um cuidado seguro tem sido um desafio constante para as organizações de saúde (CRUZ FF, et al., 2018).

De acordo com Falcão RMM, et al. (2019), a principal meta organizacional inerente à qualidade assistencial é a segurança do paciente com prevenção máxima do episódio de eventos adversos, definidos como lesões ou danos não propositados que derivam em incapacidade ou disfunção de magnitude diversa, temporária ou permanente, e/ou prolongamento do tempo de permanência no serviço de saúde (OLIVEIRA RM, et al., 2014).

Contudo, dentre os eventos adversos mais mencionados pelos pesquisadores, os erros de dose são um dos problemas mais frequentes relacionados à administração de medicamentos (18,52\%), intervindo na qualidade da assistência proporcionada aos pacientes hospitalizados. Esse risco é aumentado na medida em que os profissionais de saúde não são capazes de ler corretamente as prescrições, procedendo em confusão durante a dispensação, distribuição, preparo e administração. Consequentemente, um dos fatores que podem auxiliar na prevenção desses eventos é a adequada identificação do paciente, uma vez que clientes com nomes semelhantes, em mesma enfermaria ou recebendo o mesmo medicamento, porém com doses diferenciadas, podem ser simplesmente confundidos, passando a receber uma dose inadequada para o seu tratamento (SILVA AT, et al., 2016).

Cavalcante AKCB, et al. (2015), destacam que os serviços de saúde necessitam estruturar o sistema de forma segura para os profissionais não cometerem erros. Na ocorrência de um imprevisto, o importante é a assimilação de que o motivo dos erros e eventos adversos é multifatorial e que os profissionais de saúde estão suscetíveis a cometê-los quando os processos técnicos e organizacionais são complicados e mal esquematizados.

Segundo um manual de estratégias para a segurança do paciente voltadas para os profissionais, a identificação do paciente é um dos meios para garantir a qualidade e a segurança do cuidado no serviço de saúde. A diversidade de profissionais atuantes e os diversos procedimentos e tratamentos exigem que sejam estabelecidas práticas seguras de identificação do paciente. A falta ou duplicidade de elementos ou mesmo as imprecisões nos dados de cadastro do paciente podem repercutir diferentes tipos de erros nos cuidados à saúde. É responsabilidade de todos os profissionais de saúde a conferência da identificação do paciente previamente a qualquer procedimento/ tratamento, de modo que o paciente correto receba o cuidado correto. Orientar o paciente e o acompanhante quanto à pulseira que é colocada no seu braço é função de todos que prestam o cuidado seguro (BANDEIRA LE, et al., 2017).

Alguns estudos apontaram a importância da implantação de um programa completo e multidimensional para avaliar e melhorar a qualidade dos cuidados de saúde como uma prioridade institucional tendo em vista à cultura de segurança com investimento no desenvolvimento de capacidades locais, no gerenciamento de riscos e nos sistemas de informação já existentes (TOBIAS GC, et al., 2014).

Cada vez mais, as instituições de saúde necessitam ter como finalidade prioritária atender integralmente e de maneira segura as necessidades de saúde de seus pacientes, sendo muito importante que as atividades desenvolvidas por seus profissionais estejam fundamentadas em pressupostos filosóficos, científicos e metodológicos claros e apropriados para garantir um modelo assistencial de qualidade. A qualidade tem sido uma finalidade, considerando os constantes aprimoramentos das práticas que procuram a integralidade do cuidado para a satisfação das necessidades de saúde e de segurança de quem depende desses trabalhadores e de seus serviços (LEMOS GC, et al., 2018). Portanto, é indubitável a importância da educação permanente, com a finalidade de fortalecer as práticas seguras, já que o profissional de saúde melhor capacitado produz cuidado mais garantido, prevenindo o acontecimento de erros e eventos adversos (PAGANI S, et al., 2019). 
Faz-se necessário nortear a construção dos programas de monitoramento para garantir a produção de informação para a tomada de decisões e estimular o compromisso de todos com a transparência nos serviços de saúde e a responsabilização com a melhoria da segurança. Enfim, os estudos sobre segurança do paciente podem colaborar para melhoria da assistência, da qualidade e para o avanço do conhecimento científico entre os profissionais de saúde.

\section{CONSIDERAÇÕES FINAIS}

Observou-se neste estudo que a segurança do paciente, em todas as suas extensões, vem se tornando uma preocupação constante para o setor da saúde, ensejo pelo qual é um assunto que necessita ser fortemente debatido na atualidade, devido às proeminências do impacto da ocorrência dos erros e dos eventos adversos nos sistemas de saúde.

A segurança do paciente não é uma problemática individual, tampouco de uma única categoria profissional, mas fruto de um processo que envolve a necessidade da transformação institucional assim, constata-se a necessidade de se constituir a segurança do paciente nas organizações de saúde enquanto processo cultural, a fim de promover uma maior consciência de cada profissional da equipe multiprofissional que atua nas organizações.

\section{REFERÊNCIAS}

1. ARAÚJO MAN, et al. Segurança do paciente na visão de enfermeiros: uma questão multiprofissional. Enfermagem em Foco, 2017; 8(1): 52-56.

2. BANDEIRA LE, et al. Condutas de educação ao familiar para promoção da segurança da criança hospitalizada: registros da equipe multiprofissional. Revista Mineira de Enfermagem, 2017; 21(n. esp).

3. BRASIL. Ministério da Saúde. Portaria no. 1.377, de 9 de julho de 2013. Aprova os protocolos de Segurança do Paciente. Diário Oficial da União 10 jul. 2013; Seção 1.

4. BRASIL. Ministério da Saúde. Documento de referência para o Programa Nacional de Segurança do Paciente. Brasília DF: Ministério da Saúde, 2014.

5. BOHRER CD, et al. Comunicação e cultura de segurança do paciente no ambiente hospitalar: visão da equipe multiprofissional. Rev Enferm UFSM, 2016; 6(1): 50-60.

6. CAVALCANTE AKCB, et al. Cuidado seguro ao paciente: contribuições da enfermagem. Revista Cubana de Enfermería, 2015; 31(4).

7. CRUZ FF, et al. Segurança do paciente na UTI: uma revisão da literatura. Revista Científica FacMais, 2018; 12(1): 167-187.

8. DUARTE SCM, et al. Eventos adversos e segurança na assistência de enfermagem. Revista Brasileira de Enfermagem, 2015; 68(1): 144-54.

9. FALCÃO RMM, et al. Risco de quedas em pessoas idosas hospitalizadas. Revista Gaúcha de Enfermagem, 2019; 40(n. esp): e20180266.

10. FALCÃO RMM, et al. Avaliação do risco de quedas em idosos hospitalizados. Revista de Enfermagem UFPE on line, 2018; 12(3): 812-5.

11. GRIGOLETO ARL, et al. Segurança do cliente e as ações frente ao procedimento cirúrgico. Rev. Eletr. Enf. [Internet], 2011; 13(2): 347-54.

12. LEMOS GC, et al. A cultura de segurança do paciente no âmbito da enfermagem: reflexão teórica. Revista de Enfermagem do Centro-Oeste Mineiro, 2018; 8(n. esp).

13. MELLO JF, BARBOSA SFF. Cultura de segurança do paciente em terapia intensiva: recomendações da enfermagem. Texto Contexto Enferm, 2013; 22(4): 1124-33.

14. MÍRANDA AP, et al. Contribuição da enfermagem à segurança do paciente: revisão integrativa. Revista de Políticas Públicas, 2017; 16(1).

15. NASCIMENTO JC, DRAGANOV PB. História da qualidade em segurança do paciente. História da enfermagem: Revista eletrônica, 2015; 6: 299-309.

16. NUNES FDO, et al. Segurança do paciente: como a enfermagem vem contribuindo para a questão? R de Pesq: cuidado é fundamental online, 2014; 6(2): 841-847. 
17. OLIVEIRA RM, et al. Estratégias para promover segurança do paciente: da identificação dos riscos à práticas baseadas em evidências. Escola Anna Nery Revista de Enfermagem, 2014; 18(1): 122-129.

18. OLIVEIRA JS, et al. Fatores extrínsecos para risco de quedas de idosos hospitalizados. Revista Enfermagem UFPE online, 2018; 12(7): 1835-40.

19. PAGANI S, et al. Cultura de segurança do paciente: avaliação de enfermeiros. Revista RENE, 2019; 20 (n. esp).

20. PEDRO DRC, et al. Violência ocupacional na equipe de enfermagem: análise à luz do conhecimento produzido. Revista Saúde debate, 2017; 41(113).

21. PERES MA, et al. Percepção de familiares e cuidadores quanto à segurança do paciente em unidades de internação pediátrica. Revista Gaúcha de Enfermagem, 2018; 39(n. esp).

22. REIS GAX, et al. Implantação das estratégias de segurança do paciente: percepções de enfermeiros gestores. Texto Contexto-Enferm, 2017; 26(2).

23. SANTOS RP, et al. Avaliação da implantação de um núcleo de segurança do paciente. Revista de Enfermagem UFPE online, 2019; 13(2): 532-537.

24. SILVA ACA, et al. A segurança do paciente em âmbito hospitalar: revisão integrativa da literatura. Cogitare Enfermagem, 2016; 21 (n. esp): 1-9.

25. SILVA AT, et al. Assistência de enfermagem e o enfoque da segurança do paciente no cenário brasileiro. Revista Saúde Debate, 2016; 40(111): 292-301.

26. SILVA AT, et al. Segurança do paciente e a atuação do enfermeiro em Hospitalar. Revista de Enfermagem UFPE online, 2018; 12(6): 1532-1538.

27. SOUZA LO, et al. O uso de protocolos de segurança do paciente nas instituições hospitalares. Rev. Bras. Pesq. Saúde, 2017; 19(1): 70-77.

28. TOBIAS GC, et al. Cultura de segurança do paciente em instituições de saúde: um estudo bibliométrico. Enfermería Global, 2014; 13(1): 336-361.

29. TOSO GL, et al. Cultura de segurança do paciente em instituições hospitalares na perspectiva da enfermagem. Revista Gaúcha de Enfermagem, 2016; 37(4): e58662.

30. WHITE GD, et al. Blame the Patient, Blame the Doctor or Blame the System? A Meta-Synthesis of Qualitative Studies of Patient Safety in Primary Care. PLoS One., 2015; 10(8): 1-42. 